\title{
Molecular and Morphological Characterization of Aromatic Farmer's Rice Varieties Collected from Different Districts of Madhya Pradesh, India
}

\author{
G. K. Koutu, Arpita Shrivastava and Yogendra Singh*
}

Department of Plant Breeding and Genetics, Jawaharlal Nehru Krishi Vishwa Vidyalaya, Jabalpur, M.P., India

*Corresponding author

\section{A B S T R A C T}

Keywords

straw $(76.56 \%)$, purple $(10.94 \%)$, gold $(7.81 \%)$ and $4.69 \%)$, aromatic rice

Article Info

Accepted:

15 December 2019 Available Online: 20 January 2020
In the present study, sixty four aromatic landraces of rice collected from different districts of Madhya Pradesh were analysed for genetic variation using SSR markers. Specially, the objective of the study was DNA fingerprinting and genetic diversity analysis of aromatic landraces to requisite to study the unique feature and to identify the duplication among them and to assist in broadening the germplasm base of future aromatic rice breeding programs. Very high variation was observed for the trait sterile lemma colour in the classes of straw $(76.56 \%)$, purple $(10.94 \%)$, gold $(7.81 \%)$ and $4.69 \%)$. Similar trend was also observed for grain weight of 1000 fully developed grains in the classes of low (28.13\%), medium (25.00\%), very high $(20.31 \%)$, very low (18.75\%) and high (7.81). The experiment for analysing genetic relationship using 31 SSR primers revealed that all primers showed polymorphism with reproducible and informative profiles. The DNA amplification pattern revealed that a total number of 594 SSR loci were amplified with an average of 19.31 loci per primer.

\section{Introduction}

Rice (Oryza sativa L.) is a staple food crop and a primary food source for more than a third of world's population. Among all the Asian countries, India occupies the largest area under cultivation and accounting for about $20 \%$ of all world rice production..Aromatic rice forms a separate group and, is nature's gift exclusive to Indian sub-continent (Glaszman 1987). Aromatic rice is known for its characteristic fragrance when cooked due to chemical 2-acetyl-1-pyroline. The aromatic landraces found in Madhya Pradesh constitute a special group of rice that is known for the best in quality. Landrace refers to domesticated plants adapted to the natural and cultural environment in which they 
live (or originated) and, in some cases, work. Landraces have been shown to be excellent sources of genes for novel alleles (McCouch et al., 1997; Hoisington et al., 1999). No systematic studies have been done for characterization of these aromatic landraces and no clear cut identification features of these landraces are yet known. Since at different places, the same landrace is grown with different names. Many farmers bring some varieties from distant places and start cultivating them with local names. So, Genetic study of these aromatic landraces is the pre requisite to study the unique feature and to identify the duplication among them.

The molecular and phenotypic characterization could reveal their phylogeny and this information would be quite useful for identification of diagnostic traits. The uniqueness of a particular variety is to be established by the test called DUS (Subba Rao et al., 2013). DUS test has been established to be the foundation of plant variety protection and also to identify a new variety from reference collection (Kwon et al., 2005). Requirement of DUS is assessed on the basis of characteristics. The characteristics are a feature of whole plant or part of plant. Moreover, the existing methods are time consuming, which have altogether led to more necessity for developing a substitutionary, less costly system. Thus, the studies on the use of molecular markers in DUS testing proving the expected capability of molecular markers have encouraged International Union for the Protection of New Varieties of Plants (UPOV) to contemplate the introduction of molecular markers to the DUS testing system (Pourabed et al., 2015).

Molecular Marker based Genetic Diversity Analysis has the potential for assessing changes in genetic diversity over time and space (Duwick, 1984). DNA markers are predominantly used in molecular characterization and diversity studies due to their abundance and repeatability (McCouch et al., 1997). Several molecular marker techniques viz. Restriction Fragment Length Polymorphism (RFLP), Random Amplified Polymorphic DNA (RAPD), Simple Sequence Repeats (SSRs), Amplified Fragment Length Polymorphism (AFLP) and Single Nucleotide Polymorphisms (SNPs) have been used to assess genetic diversity of various rice cultivars throughout the world (Joshi et al., 2000). They are more reliable and remain unaffected across growth stages, seasons, locations and agronomic practices. These markers have been recently utilized for many purposes including genome mapping, gene tagging, estimation of genetic diversity, varietal differentiation, resolution of uncertain parentage and purity testing (Olufowote et al., 1997; Coburn et al., 2002; Ni et al., 2002, Singh,et al., 2008, Singh and Singh,2012). Among different PCR based markers, the microsatellite markers based on simple sequence repeats (SSRs) are preferred over other molecular markers due to their ease of application, high reproducibility, rapid analysis, low cost, easy scoring patterns and greater allelic diversity (Chen et al., 1997).

More than 2200 microsatellite markers have been mapped to specific locations in rice genome (Mc Couch et al., 2002). A random set of these mapped markers providing genome wide coverage should facilitate an unbiased assay of genetic diversity and thus giving a robust, unambiguous molecular description of rice cultivars (Nagraju et al., 2002).

In the present study, sixty four aromatic landraces of rice collected from different districts of Madhya Pradesh were analyzed for genetic variation using SSR markers. Specially, the objective of the study was DNA fingerprinting and genetic diversity analysis of aromatic landraces to requisite to study the 
unique feature and to identify the duplication among them and to assist in broadening the germplasm base of future aromatic rice breeding programs.

\section{Materials and Methods}

\section{Germplasm Collection and Morphological Characterization}

A total of sixty four farmers varieties collected from different districts of Madhya Pradesh (Table 1) were grown in a randomized complete block design with three replications at Seed Breeding Farm, JNKVV Jabalpur in kharif 2015 and kharif 2016, each entry was sown in three rows of $2 \mathrm{~m}$ length at spacing of $20 \mathrm{~cm}$ between rows and $15 \mathrm{~cm}$ between plants. Crop was raised following recommended package of practices. Observations were recorded on five randomly chosen plants of each genotype per replication for twenty five traits, according to the National Test Guidelines for DUS test in rice which was developed by Directorate of Rice Research, Rajendarnagar, Hyderabad (Shobha Rani et al., 2004). The observation of various characteristics was recorded at different stages of growth with appropriate procedures as per the DUS test guidelines of PPV \& FR Act, 2001.

\section{DNA Extraction}

Total genomic DNA was isolated from fresh young leaves of 64 farmers varieties collected from different districts of Madhya Pradesh following the CTAB (cetyl trimethyl ammonium bromide) procedure as described by Saghai Maroof et al., (1984) with some modifications. Quantification of DNA was accomplished by analyzing the DNA on $0.8 \%$ agarose gel stained with ethidium bromide using diluted uncut lambda DNA as standard. Final concentration was adjusted to $50 \mathrm{ng}^{-1} \mathrm{l}^{-1}$ for further uses in PCR analysis.

\section{PCR amplification}

A total of 31 SSRs primer pairs, distributed across the genome of rice were used (www.gramenae.org.in). The details of SSR markers, their sequences and motifs are given in table 2. DNA was amplified by PCR using our previously standardized method (Sahu et al., 2012) in a total volume of $10 \mu \mathrm{l}$ containing 2X PCR assay buffer, $1.5 \mathrm{mM} \mathrm{MgCl}_{2}, 100 \mu \mathrm{M}$ of each dNTPs, 12ng each of forword and reverse primers, 0.2 units of Taq DNA polymerase and $25 \mathrm{ng}$ of genomic DNA template. Amplification reaction initiated with a 5-minute pre-denaturation steps at $94^{\circ} \mathrm{C}$ followed by 35 cycles of DNA denaturation at $94^{0} \mathrm{C}$ for 30 seconds, primer annealing at 50$55^{\circ} \mathrm{C}$ for 30 seconds and DNA extension at $72^{0} \mathrm{C}$ for 7 minutes was performed after 35 cycles. Amplified PCR products was separated on $2.0 \%$ of agarose gel at a volage of $90 \mathrm{~V}$ for the period of 45 minutes to 1 hour in $1 \mathrm{X}$ TBE buffer stained with ethidium bromide. The gel was visualized in UV transilluminator and photograph taken using Alpho Digidoc gel documentation instrument.

\section{SSR allele scoring and data analysis}

The presence or absence of SSR fragment in each accession was recorded for all the polymorphic SSR markers. The SSR bands appearing without ambiguity were scored as 1 (present) and 0 (absent) for each primer. The size of the amplified product was calculated on the basis of its mobility relative to molecular mass of marker (100 bp DNA ladder). The genetic similarity among genotypes was estimated based on Jaccard's similarity coefficient. The resulting similarity matrix was further analysed using the unweighted pair-group method arithmetic average (UPGMA) clustering algorithm for construction of dendrogram; the computations were carried out using NTSYSpc version 2.2 (Rohlf 2000). 


\section{Results and Discussion}

\section{Morphological characterization of farmer's varieties}

Twenty two (88\%) out of 25 phenotypic traits showed variation among the 64 aromatic farmers varieties of rice, whereas no variation was observed for the traits like leaf: auricle, leaf: shape of ligule and stem: anthocyanin colouration of nodes. The summarised data of all the traits for farmers varieties are presented in table 2 .

Most of the farmer's varieties exhibited green $(90.63 \%)$ and light purple $(7.81 \%)$ basal leaf sheath colour. One farmers variety Nagkesar showed purple basal leaf sheath colour. Weak $(35.94 \%$ \& $85.94 \%)$ and medium $(64.06 \%$ \& v14.06\%) pubescence on blade surface and lemma of the spikelet showed by all the farmers varieties, respectively. Colourless leaf anthocyanin colouration of auricles expressed by most of the farmers varieties (93.75\%) followed by light purple $(6.25 \%)$.

White (92.19\%) and light purple (7.81\%) ligule colour exhibited by farmers varieties. Most of the farmer's varieties exhibited medium (56.25\%), late $(26.56 \%)$ and early $(17.19 \%)$ time of heading. Most of the lines showed erect $(87.50 \%$ \& $15.63 \%)$, semi erect $(12.50 \% \& 68.75 \%)$ and horizontal $(0.00 \%$ \& $15.63 \%)$ flag leaf attitude of blade in early and late observation, respectively. Lemma anthocyanin colouration is absent in most of the lines $(85.94 \%)$, while rest of the lines (14.07\%) expressed weak, medium, strong and very strong anthocyanin colouration of lemma. White stigma colour expressed by most of the lines $(84.38 \%)$ followed by purple (14.06\%) and light purple (1.56\%).

High variation observed in the character stem length (excluding panicle) in the classes of very long (39.06\%), long $(25.00 \%)$, medium $(23.44 \%)$ and short $(12.50 \%)$ and same is also for panicle length in the classes of long $(57.81 \%)$, medium $(28.13 \%)$, very long $(9.38 \%)$ and short $(4.69 \%)$. Deflexed panicle curvature exhibited by most of the lines $(85.94 \%)$ followed by drooping $(10.94 \%)$ and semi straight $(3.13 \%)$. Most of the lines had medium $(54.69 \%)$ number of panicle per plant followed by few (40.63\%) and many (4.69\%). Yellowish white colour of tip of lemma showed by most of the lines $(54.69 \%)$ followed by brown (25.00\%), black (12.50\%), white $(6.25 \%)$ and purple $(1.56 \%)$. Panicle awns present in $65.63 \%$ of the farmers varieties, out of which $69.04 \%$ showed yellowish white awn colour followed by yellowish brown $(21.42 \%)$ and reddish brown $(9.52 \%)$ and their distribution in upper half only $(50.00 \%)$, whole length (45.24\%) and tip only $(4.76 \%)$. Most of the lines expressed erect to semi erect $(78.13 \%)$ attitude of secondary branches in panicle followed by semi erect $(20.31 \%)$ and semi erect to spreading $(1.56 \%)$. Farmers varieties showed well exserted (84.38\%) and mostly exserted (15.63) type of panicle exsertion. Very high variation was observed for the trait sterile lemma colour in the classes of straw $(76.56 \%)$, purple $(10.94 \%)$, gold $(7.81 \%)$ and $4.69 \%$ ). Similar trend was also observed for grain weight of 1000 fully developed grains in the classes of low $(28.13 \%)$, medium $(25.00 \%)$, very high $(20.31 \%)$, very low (18.75\%) and high (7.81). 
Table.1 DUS Characterization of aromatic cultivars collected from different districts of Madhya Pradesh

\begin{tabular}{|c|c|c|c|c|c|c|c|c|c|c|c|c|c|c|c|c|c|c|c|c|c|c|c|c|c|c|c|}
\hline S no. & Cultivar & Place of collection & 2 & 8 & 9 & 10 & 14 & 15 & 20 & 21 & 22 & 25 & 27 & 29 & 30 & 33 & 34 & 35 & 36 & 37 & 39 & 40 & 42 & 45 & 46 & 49 & 50 \\
\hline 1 & Pusa Basmati & Umariya & 1 & 5 & 9 & 1 & 3 & 1 & 5 & 1 & 3 & 1 & 1 & 3 & 1 & 9 & 3 & 5 & 5 & 2 & 9 & 1 & 5 & 5 & 7 & 1 & 5 \\
\hline 2 & Badalphool & Umariya & 3 & 3 & 9 & 2 & 3 & 2 & 3 & 1 & 3 & 5 & 5 & 5 & 1 & 5 & 1 & 5 & 5 & 6 & 9 & 4 & 1 & 3 & 7 & 3 & 3 \\
\hline 3 & Luchai & Umariya & 1 & 3 & 9 & 1 & 3 & 1 & 5 & 1 & 3 & 1 & 1 & 9 & 1 & 5 & 3 & 5 & 3 & 2 & 1 & - & - & 3 & 7 & 1 & 5 \\
\hline 4 & Chinnor & Umariya & 1 & 5 & 9 & 1 & 3 & 1 & 7 & 1 & 3 & 1 & 1 & 9 & 1 & 5 & 3 & 5 & 3 & 2 & 1 & - & - & 3 & 7 & 1 & 1 \\
\hline 5 & Jeeraphool & Umariya & 1 & 5 & 9 & 1 & 3 & 1 & 7 & 1 & 3 & 1 & 1 & 7 & 1 & 5 & 3 & 5 & 5 & 2 & 1 & - & - & 3 & 7 & 1 & 1 \\
\hline 6 & Basmatiya & Umariya & 1 & 3 & 9 & 1 & 3 & 1 & 3 & 1 & 3 & 1 & 1 & 5 & 1 & 5 & 1 & 5 & 3 & 2 & 9 & 1 & 3 & 3 & 7 & 1 & 3 \\
\hline 7 & Badalphool & Umariya & 3 & 3 & 9 & 2 & 3 & 2 & 3 & 1 & 3 & 5 & 5 & 5 & 1 & 5 & 1 & 5 & 5 & 6 & 9 & 4 & 1 & 3 & 7 & 3 & 3 \\
\hline 8 & Karhani & Umariya & 1 & 3 & 9 & 1 & 3 & 1 & 3 & 1 & 5 & 1 & 1 & 5 & 1 & 3 & 1 & 3 & 5 & 2 & 9 & 1 & 5 & 3 & 5 & 1 & 9 \\
\hline 9 & Sukaraphool & Shahdol & 1 & 3 & 9 & 1 & 3 & 1 & 5 & 1 & 3 & 9 & 1 & 9 & 1 & 7 & 3 & 7 & 3 & 5 & 9 & 4 & 5 & 3 & 7 & 4 & 5 \\
\hline 10 & Kapoorsar & Shahdol & 1 & 5 & 9 & 1 & 3 & 1 & 5 & 3 & 5 & 9 & 1 & 9 & 1 & 9 & 5 & 7 & 3 & 6 & 1 & - & - & 3 & 7 & 4 & 3 \\
\hline 11 & Vishnubhog & Shahdol & 1 & 5 & 9 & 1 & 3 & 1 & 5 & 1 & 3 & 1 & 1 & 9 & 1 & 7 & 3 & 5 & 5 & 3 & 9 & 1 & 3 & 3 & 7 & 2 & 3 \\
\hline 12 & Lohandi Choti & Shahdol & 1 & 3 & 9 & 1 & 3 & 1 & 5 & 3 & 3 & 1 & 1 & 9 & 1 & 7 & 3 & 5 & 3 & 3 & 1 & - & - & 3 & 7 & 1 & 3 \\
\hline 13 & Vishnubhog & Shahdol & 1 & 5 & 9 & 1 & 3 & 1 & 5 & 1 & 3 & 1 & 1 & 9 & 1 & 7 & 3 & 5 & 5 & 3 & 9 & 2 & 3 & 3 & 7 & 2 & 3 \\
\hline 14 & Kandha Jal & Shahdol & 1 & 3 & 9 & 1 & 3 & 1 & 5 & 1 & 5 & 1 & 1 & 7 & 1 & 5 & 3 & 5 & 5 & 2 & 9 & 1 & 3 & 7 & 7 & 1 & 7 \\
\hline 15 & Jeeraphool & Shahdol & 1 & 5 & 9 & 1 & 3 & 1 & 7 & 1 & 3 & 1 & 1 & 7 & 1 & 5 & 3 & 5 & 5 & 2 & 1 & - & - & 3 & 7 & 1 & 1 \\
\hline 16 & Laxmibhog & Dindori & 1 & 3 & 9 & 1 & 3 & 1 & 5 & 1 & 5 & 1 & 1 & 9 & 1 & 7 & 5 & 5 & 3 & 3 & 1 & - & - & 3 & 7 & 1 & 1 \\
\hline 17 & Kshatriya & Dindori & 1 & 5 & 9 & 1 & 3 & 1 & 7 & 1 & 3 & 1 & 1 & 9 & 1 & 7 & 3 & 5 & 3 & 2 & 9 & 2 & 5 & 3 & 7 & 1 & 3 \\
\hline 18 & Khuddy & Dindori & 1 & 5 & 9 & 1 & 3 & 1 & 7 & 1 & 5 & 1 & 1 & 9 & 1 & 7 & 5 & 5 & 3 & 3 & 1 & - & - & 3 & 7 & 3 & 5 \\
\hline 19 & Luchai & Dindori & 1 & 3 & 9 & 1 & 3 & 1 & 5 & 1 & 3 & 1 & 1 & 9 & 1 & 5 & 3 & 5 & 3 & 2 & 1 & - & - & 3 & 7 & 1 & 5 \\
\hline 20 & Kodo Kapoor & Dindori & 1 & 5 & 9 & 1 & 3 & 1 & 5 & 3 & 3 & 1 & 1 & 9 & 1 & 7 & 5 & 7 & 3 & 3 & 1 & - & - & 3 & 7 & 1 & 1 \\
\hline 21 & Shrikamal & Dindori & 1 & 5 & 9 & 1 & 3 & 1 & 7 & 1 & 3 & 5 & 1 & 9 & 1 & 9 & 5 & 7 & 3 & 6 & 1 & - & - & 5 & 7 & 4 & 1 \\
\hline 22 & Chota Luchai & Dindori & 1 & 3 & 9 & 1 & 3 & 1 & 5 & 1 & 3 & 1 & 1 & 7 & 1 & 3 & 5 & 5 & 5 & 1 & 1 & - & - & 3 & 7 & 1 & 7 \\
\hline 23 & Vishnubhog & Dindori & 1 & 5 & 9 & 1 & 3 & 1 & 5 & 3 & 3 & 1 & 1 & 9 & 1 & 7 & 3 & 5 & 5 & 3 & 9 & 2 & 3 & 3 & 7 & 2 & 3 \\
\hline 24 & Jeerashankar & Mandla & 1 & 5 & 9 & 1 & 3 & 1 & 7 & 1 & 3 & 3 & 1 & 9 & 1 & 9 & 3 & 7 & 5 & 6 & 1 & - & - & 3 & 7 & 4 & 1 \\
\hline 25 & Ranikajar & Mandla & 3 & 3 & 9 & 1 & 3 & 2 & 3 & 1 & 3 & 7 & 5 & 3 & 1 & 5 & 3 & 5 & 5 & 6 & 9 & 4 & 5 & 3 & 5 & 4 & 3 \\
\hline 26 & Akash & Mandla & 1 & 3 & 9 & 1 & 3 & 1 & 3 & 1 & 5 & 1 & 1 & 3 & 1 & 7 & 3 & 5 & 5 & 2 & 1 & - & - & 3 & 5 & 1 & 5 \\
\hline 27 & Lakha Luchai & Mandla & 1 & 3 & 9 & 1 & 3 & 1 & 5 & 1 & 3 & 1 & 1 & 7 & 1 & 5 & 3 & 5 & 3 & 3 & 1 & - & - & 3 & 7 & 1 & 5 \\
\hline 28 & Chota Luchai & Mandla & 1 & 3 & 9 & 1 & 3 & 1 & 5 & 1 & 3 & 1 & 1 & 7 & 1 & 3 & 5 & 5 & 5 & 1 & 1 & - & - & 3 & 7 & 1 & 7 \\
\hline 29 & Durgawati & Mandla & 3 & 3 & 9 & 1 & 3 & 2 & 3 & 1 & 3 & 1 & 5 & 7 & 1 & 7 & 3 & 5 & 3 & 1 & 9 & 1 & 5 & 3 & 7 & 1 & 5 \\
\hline 30 & Mahuadheta & Mandla & 1 & 5 & 9 & 2 & 3 & 1 & 5 & 1 & 3 & 1 & 5 & 9 & 1 & 7 & 1 & 5 & 3 & 3 & 9 & 2 & 3 & 3 & 7 & 1 & 9 \\
\hline 31 & Mahuadheta & Mandla & 1 & 5 & 9 & 1 & 3 & 1 & 5 & 1 & 3 & 1 & 5 & 9 & 1 & 7 & 1 & 5 & 3 & 3 & 9 & 2 & 3 & 3 & 7 & 1 & 9 \\
\hline 32 & Durgawati & Mandla & 3 & 3 & 9 & 1 & 3 & 2 & 3 & 1 & 3 & 1 & 5 & 7 & 1 & 7 & 3 & 5 & 5 & 1 & 9 & 1 & 5 & 3 & 7 & 1 & 5 \\
\hline 33 & Nagkesar & Mandla & 4 & 5 & 9 & 2 & 3 & 3 & 3 & 1 & 5 & 1 & 4 & 7 & 1 & 7 & 1 & 5 & 3 & 3 & 1 & - & - & 3 & 7 & 1 & 7 \\
\hline 34 & Nungi & Mandla & 1 & 5 & 9 & 3 & 3 & 1 & 3 & 1 & 5 & 1 & 5 & 7 & 1 & 5 & 3 & 5 & 3 & 3 & 9 & 2 & 3 & 3 & 5 & 1 & 9 \\
\hline 35 & CSR 30 & Hosangabad & 1 & 5 & 9 & 1 & 3 & 1 & 5 & 1 & 3 & 1 & 1 & 7 & 1 & 7 & 5 & 5 & 5 & 2 & 9 & 1 & 5 & 3 & 5 & 1 & 5 \\
\hline 36 & Pusa 1121 & Hosangabad & 1 & 5 & 9 & 1 & 3 & 1 & 5 & 1 & 3 & 1 & 1 & 5 & 1 & 7 & 3 & 5 & 7 & 2 & 9 & 1 & 3 & 5 & 7 & 1 & 9 \\
\hline
\end{tabular}




\begin{tabular}{|c|c|c|c|c|c|c|c|c|c|c|c|c|c|c|c|c|c|c|c|c|c|c|c|c|c|c|c|}
\hline 37 & CSR 30 & Hosangabad & 1 & 5 & 9 & 1 & 3 & 1 & 5 & 1 & 3 & 1 & 1 & 7 & 1 & 7 & 5 & 5 & 5 & 2 & 9 & 1 & 5 & 3 & 5 & 1 & 5 \\
\hline 38 & Pusa 1121 & Hosangabad & 1 & 5 & 9 & 1 & 3 & 1 & 5 & 1 & 3 & 1 & 1 & 5 & 1 & 7 & 3 & 5 & 5 & 2 & 9 & 1 & 3 & 5 & 7 & 1 & 9 \\
\hline 39 & Pusa 1 & Hosangabad & 1 & 5 & 9 & 1 & 3 & 1 & 5 & 1 & 3 & 1 & 1 & 5 & 1 & 7 & 3 & 5 & 5 & 2 & 9 & 1 & 5 & 3 & 7 & 1 & 5 \\
\hline 40 & Menka & Hosangabad & 1 & 5 & 9 & 1 & 3 & 1 & 5 & 3 & 3 & 1 & 1 & 3 & 1 & 7 & 3 & 5 & 3 & 2 & 9 & 1 & 3 & 5 & 5 & 1 & 9 \\
\hline 41 & Desi Basmati & Hosangabad & 1 & 5 & 9 & 1 & 3 & 1 & 7 & 1 & 3 & 1 & 1 & 7 & 1 & 7 & 5 & 5 & 5 & 3 & 9 & 1 & 3 & 3 & 7 & 2 & 3 \\
\hline 42 & Pusa 1121 & Hosangabad & 1 & 5 & 9 & 1 & 3 & 1 & 5 & 1 & 3 & 1 & 1 & 5 & 1 & 7 & 3 & 5 & 7 & 2 & 9 & 1 & 3 & 5 & 7 & 1 & 9 \\
\hline 43 & Sugandha & Raisen & 1 & 5 & 9 & 1 & 3 & 1 & 5 & 1 & 3 & 1 & 1 & 3 & 1 & 7 & 3 & 5 & 3 & 2 & 9 & 1 & 3 & 5 & 5 & 1 & 9 \\
\hline 44 & Pusa 1121 & Raisen & 1 & 5 & 9 & 1 & 3 & 1 & 5 & 1 & 3 & 1 & 1 & 5 & 1 & 7 & 3 & 5 & 5 & 2 & 9 & 1 & 3 & 5 & 7 & 1 & 9 \\
\hline 45 & Pusa 1 & Raisen & 1 & 5 & 9 & 1 & 3 & 1 & 5 & 1 & 3 & 1 & 1 & 5 & 1 & 7 & 3 & 5 & 5 & 2 & 9 & 1 & 5 & 3 & 7 & 1 & 5 \\
\hline 46 & Pusa 1121 & Raisen & 1 & 5 & 9 & 1 & 3 & 1 & 5 & 1 & 3 & 1 & 1 & 5 & 1 & 7 & 3 & 5 & 5 & 2 & 9 & 1 & 3 & 5 & 7 & 1 & 9 \\
\hline 47 & Kshatriya & Raisen & 1 & 5 & 9 & 1 & 3 & 1 & 7 & 1 & 3 & 1 & 1 & 9 & 1 & 7 & 3 & 5 & 3 & 2 & 9 & 2 & 5 & 3 & 7 & 1 & 3 \\
\hline 48 & Pusa 1121 & Raisen & 1 & 5 & 9 & 1 & 3 & 1 & 5 & 1 & 3 & 1 & 1 & 5 & 1 & 7 & 3 & 5 & 5 & 2 & 9 & 1 & 3 & 5 & 5 & 1 & 9 \\
\hline 49 & Pusa 1121 & Raisen & 1 & 5 & 9 & 1 & 3 & 1 & 5 & 1 & 3 & 1 & 1 & 5 & 1 & 7 & 3 & 5 & 5 & 2 & 9 & 1 & 3 & 5 & 7 & 1 & 5 \\
\hline 50 & Pusa 1 & Raisen & 1 & 5 & 9 & 1 & 3 & 1 & 5 & 1 & 3 & 1 & 1 & 5 & 1 & 7 & 3 & 5 & 5 & 2 & 9 & 1 & 5 & 3 & 7 & 1 & 5 \\
\hline 51 & Menka & Raisen & 1 & 5 & 9 & 1 & 3 & 1 & 5 & 3 & 3 & 1 & 1 & 3 & 1 & 7 & 3 & 5 & 3 & 2 & 9 & 1 & 3 & 5 & 5 & 1 & 9 \\
\hline 52 & Pusa 1 & Raisen & 1 & 5 & 9 & 1 & 3 & 1 & 5 & 1 & 3 & 1 & 1 & 5 & 1 & 7 & 3 & 5 & 5 & 2 & 9 & 1 & 5 & 3 & 7 & 1 & 5 \\
\hline 53 & Kshatirya & Katni & 1 & 5 & 9 & 1 & 3 & 1 & 7 & 1 & 3 & 1 & 1 & 9 & 1 & 7 & 3 & 5 & 3 & 2 & 9 & 2 & 5 & 3 & 7 & 1 & 3 \\
\hline 54 & Madhuri & Katni & 1 & 3 & 9 & 1 & 3 & 1 & 5 & 1 & 3 & 1 & 1 & 3 & 1 & 5 & 1 & 5 & 5 & 2 & 9 & 1 & 5 & 3 & 7 & 1 & 3 \\
\hline 55 & Dubraj & Katni & 1 & 3 & 9 & 1 & 3 & 1 & 7 & 1 & 3 & 1 & 1 & 7 & 1 & 5 & 3 & 5 & 3 & 3 & 9 & 1 & 5 & 3 & 7 & 1 & 3 \\
\hline 56 & Vishnubhog & Balaghat & 1 & 5 & 9 & 1 & 3 & 1 & 5 & 3 & 3 & 1 & 1 & 9 & 1 & 7 & 3 & 5 & 5 & 3 & 9 & 2 & 3 & 3 & 7 & 2 & 3 \\
\hline 57 & Jeerashankar & Balaghat & 1 & 5 & 9 & 1 & 3 & 1 & 7 & 1 & 3 & 3 & 1 & 9 & 1 & 9 & 3 & 7 & 5 & 6 & 1 & - & - & 3 & 7 & 4 & 1 \\
\hline 58 & Chinnor & Balaghat & 1 & 5 & 9 & 1 & 3 & 1 & 7 & 1 & 3 & 1 & 1 & 9 & 1 & 5 & 3 & 5 & 3 & 2 & 1 & - & - & 3 & 7 & 1 & 1 \\
\hline 59 & Makram & Balaghat & 1 & 3 & 9 & 1 & 3 & 1 & 5 & 3 & 3 & 1 & 1 & 3 & 1 & 5 & 1 & 5 & 7 & 2 & 9 & 1 & 3 & 3 & 7 & 1 & 3 \\
\hline 60 & Chinnor Desi & Balaghat & 1 & 3 & 9 & 1 & 3 & 1 & 7 & 1 & 3 & 1 & 1 & 9 & 1 & 7 & 3 & 5 & 5 & 2 & 9 & 1 & 5 & 3 & 7 & 1 & 1 \\
\hline 61 & Dubraj & Balaghat & 1 & 3 & 9 & 1 & 3 & 1 & 7 & 1 & 3 & 1 & 1 & 7 & 1 & 5 & 3 & 5 & 5 & 3 & 9 & 1 & 5 & 3 & 7 & 1 & 1 \\
\hline 62 & Jeerashankar & Balaghat & 1 & 5 & 9 & 1 & 3 & 1 & 7 & 1 & 3 & 3 & 1 & 9 & 1 & 9 & 3 & 7 & 5 & 6 & 1 & - & - & 3 & 7 & 4 & 3 \\
\hline 63 & Chinnor & Balaghat & 1 & 5 & 9 & 1 & 3 & 1 & 7 & 1 & 3 & 1 & 1 & 9 & 1 & 5 & 3 & 5 & 3 & 2 & 1 & - & - & 3 & 7 & 1 & 1 \\
\hline 64 & Tulsi & Balaghat & 1 & 3 & 9 & 1 & 3 & 1 & 3 & 1 & 5 & 1 & 5 & 7 & 1 & 7 & 1 & 3 & 5 & 2 & 1 & - & - & 5 & 7 & 1 & 7 \\
\hline
\end{tabular}

2-Basal leaf: sheath colour, 8- Leaf: pubescence of blade surface, 9- Leaf: auricles, 10- Leaf: anthocyanin colouration of auricles, 14- Leaf: shape of ligule, 15- Leaf: colour of ligule, 20-Time of heading, 21- Flag leaf attitude of blade (early observation), 22- Spikelet: density of pubescence of lemma, 25- Lemma: anthocyanin colouration of area below apex, 27- Spikelet: colour of stigma, 29- Stem: length (excluding panicle), 27- Stem: anthocyanin colouration of nodes, 28- Stem: intensity of anthocyanin colouration of nodes, 30-Stem: anthocyanin colouration of nodes, 33- Panicle: length of main axis (cm), 34- Flag leaf attitude of blade (late observation), 35- Panicle: curvature of main axis, 36-Panicle: number per plant, 37- Spikelet: colour of tip of lemma, 39- Panicle: awns, 40- Panicle: colour of awns, 42- Panicle: distribution of awns, 45- Panicle: attitude of branches, 46- Panicle: exsertion, 49- Sterile lemma colour, 50- Grain weight of 1000 fully developed grains. 
Table.2 SSR markers with their sequences selected for the study

\begin{tabular}{|c|c|c|c|}
\hline Primers & Reverse sequence & Forward sequence & Amplification temperature $\left({ }^{\circ} \mathrm{C}\right)$ \\
\hline RM 1 & GCGTTGGTTGGACCTGAC & GCGAAAACACAATGCAAAAA & 55 \\
\hline RM 5 & GCATCCGATCTTGATGGG & TGCAACTTCTAGCTGCTCGA & 55 \\
\hline RM 8 & AACACAGCAGGTACGCGC & CGCTAGGGCAGCATCTAAA & 55 \\
\hline RM 11 & ATAGCGGGCGAGGCTTAG & TCTCCTCTTCCCCCGATC & 55 \\
\hline RM 16 & AACACAGCAGGTACGCGC & CGCTAGGGCAGCATCTAAA & 55 \\
\hline RM 17 & GGTGATCCTTTCCCATTTCA & TGCCCTGTTATTTTCTTCTCTC & 55 \\
\hline RM 30 & TCACCTCACCACACGACACG & GGTTAGGCATCGTCACGG & 55 \\
\hline RM 137 & CGGGTGGTCCCCGAGGATCTTG & GACATCGCCACCAGCCCACCAC & 55 \\
\hline RM 152 & CCGTAGACCTTCTTGAAGTAG & GAAACCACCACACCTCACCG & 55 \\
\hline RM 154 & СТCCTCCTCCTGCGACCGCTCC & ACCCTCTCCGCCTCGCCTCCTC & 55 \\
\hline RM 201 & CTACCTCCTTTCTAGACCGATA & CTCGTTTATTACCTACAGTACC & 55 \\
\hline RM 202 & CCAGCAAGCATGTCAATGTA & CAGATTGGAGATGAAGTCCTCC & 55 \\
\hline RM 217 & GGGTGTGAACAAAGACAC & ATCGCAGCAATGCCTCGT & 55 \\
\hline RM 219 & CATATCGGCATTCGCCTG & CGTCGGATGATGTAAAGCCT & 55 \\
\hline RM 223 & GAAGGCAAGTCTTGGCACTG & GAGTGAGCTTGGGCTGAAAC & 55 \\
\hline RM 228 & GCTTGCGGCTCTGCTTAC & CTGGCCATTAGTCCTTGG & 55 \\
\hline RM 231 & CACTTGCATAGTTCTGCATTG & CCAGATTATTTCCTGAGGTC & 55 \\
\hline RM 234 & CACGTGAGACAAAGACGGAG & ACAGTATCCAAGGCCCTGG & 55 \\
\hline
\end{tabular}


Int.J.Curr.Microbiol.App.Sci (2020) 9(1): 124-135

\begin{tabular}{|c|c|c|c|}
\hline RM 237 & TGGGAAGAGAGCACTACAGC & CAAATCCCGACTGCTGTCC & 55 \\
\hline RM 242 & TATATGCCAAGACGGATGGG & GGCCAACGTGTGTATGTCTC & 55 \\
\hline RM 251 & ATGCGGTTCAAGATTCGATC & GAATGGCAATGGCGCTAG & 55 \\
\hline RM 256 & GTTGATTTCGCCAAGGGC & GACAGGGAGTGATTGAAGGC & 55 \\
\hline RM 260 & GAACAATCCCTTCTACGATCG & ACTCCACTATGACCCAGAG & 55 \\
\hline RM 263 & GCTACGTTTGAGCTACCACG & CCCAGGCTAGCTCATGAACC & 55 \\
\hline RM 271 & TCGGTGAGACCTAGAGAGCC & TCAGATCTACAATTCCATCC & 55 \\
\hline RM 25 & CTACCATCAAAACCAATGTTC & GGAAAGAATGATCTTTTCATGG & 55 \\
\hline RM 283 & CGGCATGAGAGTCTGTGATG & GTCTACATGTACCCTTGTTGGG & 55 \\
\hline RM 338 & GGCAAACCGATCACTCAGTC & CACAGGAGCAGGAGAAGAGC & 55 \\
\hline RM 488 & TAGCAACAACCAGCGTATGC & CAGCTAGGGTTTTGAGGCTG & 55 \\
\hline RM 255 & CGAAACCGCTCAGTTCAAC & TGTTGCGTGTGGAGATGTG & 55 \\
\hline RM 277 & CAAGGCTTGCAAGGGAAG & CGGTCAAATCATCACCTGAC & 55 \\
\hline
\end{tabular}


Table.3 Number, polymorphic and unique alleles and allele size $\mathrm{n}$ involving SSR markers

\begin{tabular}{|c|c|c|c|c|c|}
\hline $\begin{array}{c}\text { S } \\
\text { no. }\end{array}$ & Primers & $\begin{array}{c}\text { Number of } \\
\text { alleles }\end{array}$ & $\begin{array}{c}\text { Polymorphic } \\
\text { alleles }\end{array}$ & $\begin{array}{c}\text { Unique } \\
\text { alleles }\end{array}$ & $\begin{array}{c}\text { Allele size range } \\
\text { (bp) }\end{array}$ \\
\hline $\mathbf{1}$ & RM 1 & 25 & 25 & 10 & $91-225$ \\
\hline $\mathbf{2}$ & RM 5 & 24 & 24 & 10 & $91-230$ \\
\hline $\mathbf{3}$ & RM 8 & 14 & 14 & 05 & $240-292$ \\
\hline $\mathbf{4}$ & RM 11 & 23 & 23 & 07 & $100-200$ \\
\hline $\mathbf{5}$ & RM 16 & 19 & 19 & 05 & $115-196$ \\
\hline $\mathbf{6}$ & RM 17 & 13 & 13 & 04 & $141-200$ \\
\hline $\mathbf{7}$ & RM 30 & 15 & 17 & 03 & $80-132$ \\
\hline $\mathbf{8}$ & RM 137 & 17 & 17 & 05 & $163-244$ \\
\hline $\mathbf{9}$ & RM 152 & 17 & 36 & 07 & $116-224$ \\
\hline $\mathbf{1 0}$ & RM 154 & 36 & 23 & 10 & $11-385$ \\
\hline $\mathbf{1 1}$ & RM 201 & 23 & 15 & 05 & $122-293$ \\
\hline $\mathbf{1 2}$ & RM 202 & 15 & 15 & 02 & $112-165$ \\
\hline $\mathbf{1 3}$ & RM 217 & 15 & 27 & 10 & $115-248$ \\
\hline $\mathbf{1 4}$ & RM 219 & 27 & 16 & 01 & $142-231$ \\
\hline $\mathbf{1 5}$ & RM 223 & 16 & 16 & 06 & $100-175$ \\
\hline $\mathbf{1 6}$ & RM 228 & 16 & 21 & 02 & $135-274$ \\
\hline $\mathbf{1 7}$ & RM 231 & 21 & 26 & 10 & $103-279$ \\
\hline $\mathbf{1 8}$ & RM 234 & 26 & 15 & 03 & $82-165$ \\
\hline $\mathbf{1 9}$ & RM 237 & 15 & 17 & 07 & $205-292$ \\
\hline $\mathbf{2 0}$ & RM 242 & 17 & 16 & 05 & $90-158$ \\
\hline $\mathbf{2 1}$ & RM 251 & 16 & 15 & 01 & $85-140$ \\
\hline $\mathbf{2 2}$ & RM 256 & 15 & 14 & 06 & $91-148$ \\
\hline $\mathbf{2 3}$ & RM 260 & 14 & 20 & 03 & $140-289$ \\
\hline $\mathbf{2 4}$ & RM 263 & 20 & 11 & 05 & $83-180$ \\
\hline $\mathbf{2 5}$ & RM 271 & 11 & 34 & 19 & $86-248$ \\
\hline $\mathbf{2 6}$ & RM 25 & 34 & 13 & 01 & $126-183$ \\
\hline $\mathbf{2 7}$ & RM 283 & 13 & 11 & 02 & $125-183$ \\
\hline $\mathbf{2 8}$ & RM 338 & 11 & 07 & $165-250$ \\
\hline $\mathbf{2 9}$ & RM 488 & 17 & 09 & $117-204$ \\
\hline $\mathbf{3 0}$ & RM 255 & 22 & 01 & $113-154$ \\
\hline $\mathbf{3 1}$ & RM 277 & 11 & & & \\
\hline & & & 11 & 03 & \\
\hline
\end{tabular}


Fig.1 SSR Profiling of Rice varieties using RM 25 SSR markers

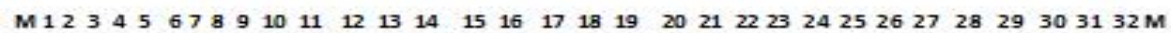

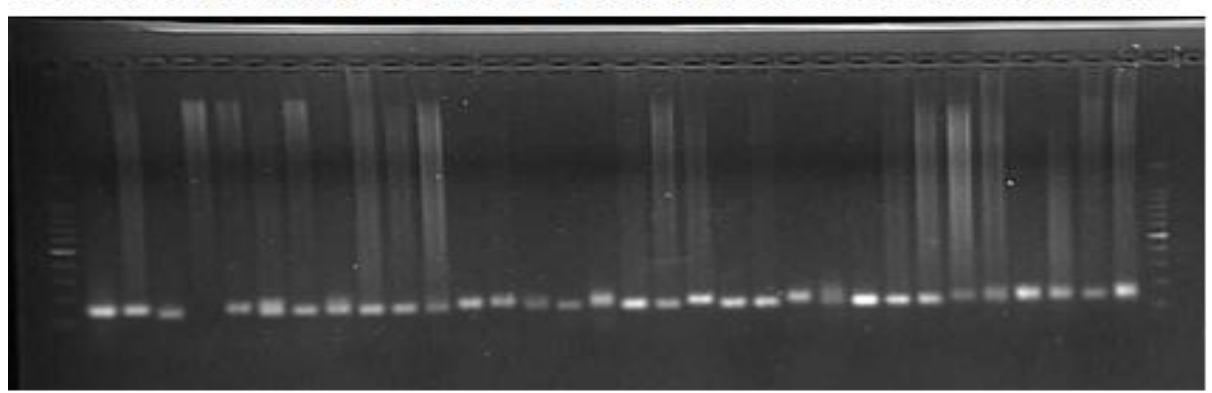

M $3334353637383940414243444546474849505152535455565758596061626364 \quad M$

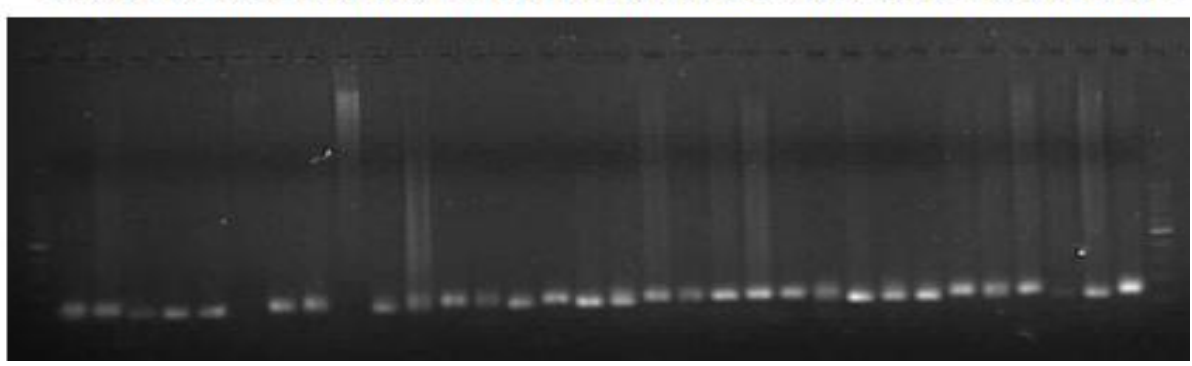

Fig.2 Unrooted Dendogram of Rice varieties based on SSR markers

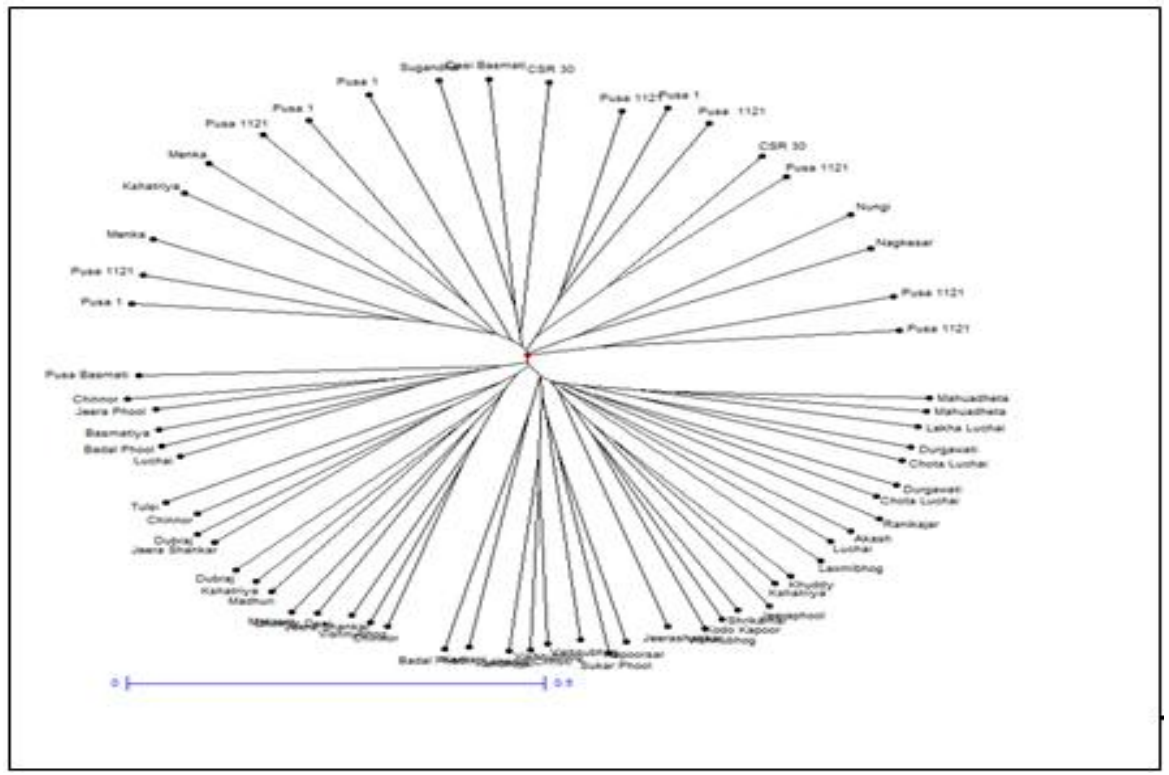




\section{Molecular characterization}

In the present investigation, 31 SSR markers were employed to assess the genetic diversity among 64 varieties of rice. The experiment for analysing genetic relationship using 31 SSR primers revealed that all primers showed polymorphism with reproducible and informative profiles (Figure-1). The DNA amplification pattern revealed that a total number of 594 SSR loci were amplified with an average of 19.31 loci per primer Table-3). Unrooted cluster analysis also depicted quiet variation among all rice varieties. (Figure-2). Molecular approaches are more reliable for assessment of genetic divergence in rice (Yogendra Singh (2011, Singh and Singh,2008): and these are being used by various workers time to time (Singh et al.,2013, Koutu et al., 2017. Koutu et al., 2019)

\section{References}

Chen X, Temnykh N, Xu Y, Cho YG and McCouch SR (1997) Development of a microsatellite framework map providing genome-wide coverage in rice (Oryza sativa L.) Theor Appl Genet 95:553-557.

Coburn JR, Temnykh SV, Paul EM and McCouch SR (2002) Design and application of microsatellite marker panels for semiautomated genotyping of rice (Oryza sativa L.). Crop Sci 42:2092-2099.

Duwick DN (. 1984). Genetic diversity in major farm crops on the farm and reserve. Econ. Bot; 32:161-178.

Ehsan Pourabed, Mohammad Reza Jazayeri Noushabadi, Seyed Hossein Jamali, Naser Moheb Alipour, Abbas Zareyan, and Leila Sadeghi (2015) Identification and DUS Testing of Rice Varieties through Microsatellite Markers. International Journal of
Plant Genomics. Volume 2015, Article ID 965073, 7 pages http://dx.doi.org/10.1155/2015/965073

Glaszmann JC (1987) Isozymes and classification of Asian rice varieties. Theor Appl Genet 74:21-30

Joshi, S. P.; Gupta, V. S.; Aggarwal, R. K.; Ranjekar, P. K. And Brar, D. S. (2000), Genetic diversity and phylogenetic relationship as revealed by inter-simple sequence repeat (ISSR) polymorphism in the genus Oryza. Theor. Appl. Genet., 100: 1311-1320.

Koutu G.K., A. Srivastava, Yogendra Singh and S. Tiwari (2019). Molecular Characterization and Genetic Diversity Assessment of Soybean Varieties using SSR Markers. International Journal of Current Microbiology and Applied Sciences. 08(04):173-182.

Koutu G.K., A. Srivastava, M.S.Bhale, Yogendra Singh S.K.Singh and D. K. Mishra (2017). DNA Fingerprinting and Genetic Divergence analysis of Rice Cultivars using SSR markers. Indian Research Journal of Genetics and Bio Technology. 09 (03): 361368

McCouch SR, Chen X, Panaud O, Temnykh S, Xu Y, Chao YG et al.,(., 1997) Microsatellite marker development, mapping and applications in rice genetics and breeding. Plant Mol. Biol; 35(1-2):89-99.

NAGARAJU, J.; KATHIRVEL, M.; KUMAR, R. Ramesh; SIDDIQ, E.A. and HASNAIN, Seyed E.(2002,) Genetic analysis of traditional and evolved Basmati and non-Basmati rice varieties by using fluorescence-based ISSR-PCR and SSR markers. Proceedings of National Academy of Sciences of the United States of America, April 30, vol. 99, no. 9, p. 5836-5841. 
Ni J, Colowit PM and Mackill D (2002) Evaluation of genetic diversity in rice subspecies using microsatellite markers. Crop Sci 42:601-607.

Olufowote JO, Xu Y, Chen X, Park WD, Beachel HM, Dilday RH, Goto M and McCouch SR (1997) Comparative evaluation of within-cultivar variation of rice (Oryza sativa L.) using microsatellite and RFLP markers. Genome 40:370-378.

SAGHAI-MAROOF, M.A. et al.,(1984.) Ribosomal DNA spacer length polymorphism in barley: Mendelian inheritance, chromosomal location and population dynamics. Proceedings of the National Academy of Sciences USA, v.81, p.8014-8018,

Rohlf, F. J. (2000). On the use of shape spaces to compare morphometric methods. Hystrix, Italian J. Mammology (n.s.), 11(1):9-25.

Singh Yogendra (2011): Molecular approaches to assess genetic divergence in Rice. GERF Bulletin of Biosciences.02(01): 41-48.

Singh Yogendra, Pani D.R, Khokhar D, Singh U S (2013). Agro-morphological
Characterization and Molecular Diversity analysis of Aromatic Rice Germplasm Using RAPD Markers. Oryza.50 (01): 26-34.

Singh Yogendra, D.R Pani, S.K Pradhan, A. Bajpai and U.S Singh (2008): "Divergence analysis for quality traits in some indigenous basmati rice genotypes". ORYZA.45(4) 263-267.

Singh Yogendra and U.S Singh (2008): "Genetic diversity analysis in Aromatic Rice Germplasm using Agro- morphological Traits. Indian Journal of plant Genetic resources.21 (1) 32-37.

Singh Yogendra and U.S.Singh (2012). Simple Sequence Repeats markers and grain quality characteristics for genetic divergence and selective identification of aromatic rices. International Journal of Advanced Biotechnology \& Research.03 (03): 711-719.

Subba Rao, L.V., Shiva Prasad, G., Chiranjivi, M., Chaitanyam U. and Surendhar, R. (2013) DUS characterization for farmer's varieties of rice. IOSR Journal of Agriculture and Veterinary Science PP 35-43.

\section{How to cite this article:}

Koutu, G. K., Arpita Shrivastava and Yogendra Singh. 2020. Molecular and Morphological Characterization of Aromatic Farmer's Rice Varieties Collected from Different Districts of Madhya Pradesh, India. Int.J.Curr.Microbiol.App.Sci. 9(01): 124-135. doi: https://doi.org/10.20546/ijcmas.2020.901.014 